\title{
3,6-二取代-1,2,4-三嗪衍生物的微波辅助合成及生物活性
}

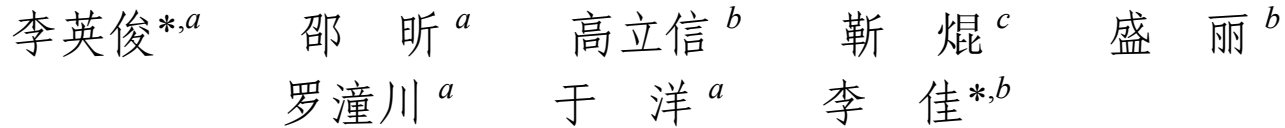 \\ ( ${ }^{a}$ 辽宁师范大学化学化工学院 大连 116029)
}

$\left({ }^{b}\right.$ 中国科学院上海药物研究所 国家新药笁选中心 药物研究国家重点实验室 上海 201203)

( ${ }^{c}$ 大连理工大学精细化工国家重点实验室 大连 116012)

\begin{abstract}
摘要 在微波辐射下, 利用 2-芳氧甲基苯并咪唑-1-乙酰肼(3)与各种(未)取代的溴代苯乙酮进行缩合, 合成出了 20 个新 的含苯并咪唑单元的 3,6-二取代-1,2,4-三嗪衍生物 4, 并利用 ${ }^{1} \mathrm{H}$ NMR, IR 和元素分析对所合成的目标化合物进行了结 构表征. 探索出了最佳合成条件. 与经典方法相比较, 该合成方法具有反应时间短、操作简便、底物范围广、易纯化、 产率较高等优点. 对所合成的目标化合物进行了 Cdc25B 和 PTP1B 抑制活性的笁选. 实验结果表明, 目标化合物 $\mathbf{4 g}$ 和 $4 \mathbf{j}$ 对 Cdc25B 表现出良好的抑制活性，目标化合物 $4 \mathbf{a}, 4 \mathbf{h}, \mathbf{4 j}, \mathbf{4 r}$ 和 $4 \mathbf{s}$ 对 PTP1B 表现出良好的抑制活性. 值得注意的是, 目标化合物 $\mathbf{j}$ 可作为用于治疗癌症和糖尿病的潜在的 Cdc25B 和 PTP1B 抑制剂.
\end{abstract}

关键词＼cjkstart微波辐射; 1,2,4-三嗪; 苯并咪唑; 合成; Cdc25B 和 PTP1B 抑制剂

\section{Microwave-Assisted Synthesis and Biological Activities of 3,6-Disubstituted-1,2,4-triazine Derivatives}

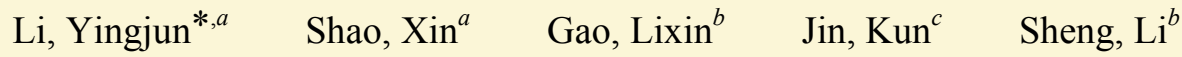 \\ Luo, Tongchuan $^{a} \quad \mathrm{Yu}, \mathrm{Yang}^{a} \quad \mathrm{Li}, \mathrm{Jia}^{*, b}$ \\ $\left({ }^{a}\right.$ College of Chemistry and Chemical Engineering, Liaoning Normal University, Dalian 116029) \\ ( ${ }^{b}$ National Center for Drug Screening, State Key Laboratory of Drug Research, Shanghai Institute of Materia Medica, \\ Chinese Academy of Sciences, Shanghai 201203) \\ ( ${ }^{c}$ State Key Laboratory of Fine Chemicals, Dalian University of Technology, Dalian 116012)
}

\begin{abstract}
Twenty new 3,6-disubstituted-1,2,4-triazine derivatives containing benzimidazole moiety (4) were synthesized via microwave-assisted condensation reactions of 2-aryloxymethylbenzimidazole-1-acetylhydrazines (3) with various (un)substituted phenacyl bromides in DMF. The structures were characterized by IR, ${ }^{1} \mathrm{H}$ NMR spectra and elemental analysis. The optimum experimental conditions were found. Compared with traditional methods, this synthetic method has such advantages as short reaction time, simple operation, broad substrate scope, easy purification and high yields. The synthesized target compounds were screened for Cdc25B and PTP1B inhibitory activities. The experimental results indicate that compounds $\mathbf{4 g}$ and $\mathbf{4 j}$ showed good inhibitory activities against $C d c 25 B$, and $\mathbf{4 a}, \mathbf{4 h}, \mathbf{4 j}, \mathbf{4 r}$ and $\mathbf{4 s}$ exhibited potent inhibitory activities against PTP1B. It is noteworthy that compound $\mathbf{4} \mathbf{j}$ can be used as potential Cdc25B and PTP1B inhibitors in the treatment of cancer and diabetes.
\end{abstract}

Keywords microwave irradiation; 1,2,4-triazines; benzimidazole; synthesis; Cdc25B and PTP1B inhibitors

1,2,4-三嗪核是一个重要的结构, 存在于许多具有 生物活性的化合物中，具有广泛的药理活性，如抗癌 ${ }^{[1]}$ 、 抗焦虑、抗炎 ${ }^{[2]}$ 、神经保护剂 ${ }^{[3]}$ 、抗真菌 ${ }^{[4]}$ 、抗癫㾁 ${ }^{[5]}$ 和降糖 ${ }^{[6]}$ 活性. 1,2,4-三嗪衍生物在农业 ${ }^{[7]}$ 和工业 ${ }^{[8]}$ 中也
有着重要的应用. 此外, $1,2,4$-三嗪核是多功能的合成 子，与许多亲二双烯体通过分子内反电子需求的 Diels-Alder 反应 ${ }^{[9]}$ 可得到大量的稠杂环体系. 因此，对 于 1,2,4-三嗪衍生物的合成和生物活性的研究引起了人

* E-mail: chemlab.lnnu@163.com

Received April 8, 2013; revised May 14, 2013; published online June 21, 2013.

Project supported by the Natural Science Foundation of Liaoning Province (No. 20102126).

辽宁省自然科学基金(No. 20102126)资助项目. 
们广泛地关注. 文献 ${ }^{[4]}$ 显示, 3-杂环取代的 1,2,4-三嗪类 化合物具有显著的生物活性. 苯并咪唑是一类重要的药 效团, 在许多天然产物及合成的具有生物活性的化合物 中都含有苯并咪唑结构 ${ }^{[10 \sim 15]}$, 因此, 在 1,2,4-三嗪的 3位上引入苯并咪唑基团是非常有意义的.

关于 1,2,4-三嗪的合成, 文献报道主要有三种典型 的方法: (1)以酰肼和 $\alpha$-卤代苯乙酮为原料, HOAc 为溶 剂, 在醋酸盐存在下, 加热回流反应制得 3,6-二取代的 1,2,4-三嗪 ${ }^{[16,17]}$. 也可采用 EtOH-DMF 为溶剂进行缩合 得到产物 ${ }^{[18]}$ ，(2) 1,2-二羰基化合物与酰胇在 $\mathrm{NH}_{4} \mathrm{OAc}$ / HOAc 中加热回流反应, 制得 3,5,6-三取代的 1,2,4-三 嗪 ${ }^{[4,9]}$, (3) 1,2-二羰基化合物与酰胺和水合肼在碱的存在 下以传统的加热方式进行反应 ${ }^{[19]}$. 然而, 这些方法仍然 有一些限制, 如反应时间长、产率适中、需要添加醋酸 盐催化剂、底物范围窄、操作复杂. 因此, 这些经典方 法已被发展, 如利用联苯甲酰与氨基胍碳酸盐在正丁醇 中的缩合 ${ }^{[20]}$ 或与氨基硫脲在 $\mathrm{H}_{2} \mathrm{O} /$ 二氧六环 ${ }^{[3]}$ 中缩合, 以 及微波辐射方法 ${ }^{[19,21 \sim 24]}$.

细胞分裂周期 25 (cell division cycle 25, 缩写 Cdc25)磷酸酯酶在真核细胞增殖的调控中起着重要作 用. 在人类细胞中, 已经确定了其三种亚型, 即 Cdc$25 \mathrm{~A}, \mathrm{Cdc} 25 \mathrm{~B}$ 和 Cdc25C. Cdc25A 和 Cdc25B 是潜在的癌 基因, 大量的人类肿瘤(乳腺癌、肺癌、大肠癌、胃癌、 前列腺癌、脑癌、卵巢癌、淋巴瘤和黑素瘤)细胞系中 存在 $\mathrm{Cdc} 25 \mathrm{~A}$ 和/或 $\mathrm{Cdc} 25 \mathrm{~B}$ 的过度表达 ${ }^{[25]}$. 在最近几年 中, 蛋白酪氨酸磷酸酯酶 1B (protein tyrosine phosphatase 1B, 缩写 PTP1B) 在人类疾病(如糖尿病、肥胖和 癌症)中具有关键的调节作用. PTP1B 是一类典型的非 跨膜蛋白酪氨酸磷酸酯酶, 在代谢中起着关键的作用, 对于 2 型糖尿病和肥胖是一种很有前途的药物靶标 ${ }^{[26]}$.

为了得到在抗癌和抗糖尿病方面具有潜在应用价 值的、选择性的、高活性的、新型的 Cdc25B 和 PTP1B 抑制剂，以及继续进行杂环化合物的有效合成和生物活 性研究的工作 ${ }^{[27,28]}$, 本课题组设计采用微波辅助法合成 一系列新的含苯并咪唑单元的 3,6-二取代-1,2,4-三嗪衍 生物、探索最佳合成条件、评价目标化合物对 Cdc25B 和 PTP1B 的抑制活性. 目标化合物合成路线见 Scheme 1.

\section{1 结果与讨论}

中间体化合物 $1 \sim 3$ 是按我们先前报道的方法 ${ }^{[28]}$ 合 成的. 其中, 中间体化合物 $\mathbf{2 b}$ 和 $\mathbf{3 b}$ 是新化合物.

由文献可知, 以酰肼和 $\alpha$-卤代苯乙酮为原料, 采用 微波辐射法制备 3,6-二取代-1,2,4三嗪时, 需要在 KOAc 存在下, 用 $\mathrm{EtOH}$ 和 $\mathrm{HOAc}$ 为溶剂进行反应 ${ }^{[23]}$, 或者用
固体无机支撑物(如硅胶等)在无溶剂条件下反应制 得 ${ }^{[24]}$. 本文采用 DMF 为溶剂, 在无醋酸盐或无机支撑 物存在下, 采用微波辐射法合成出了目标化合物.
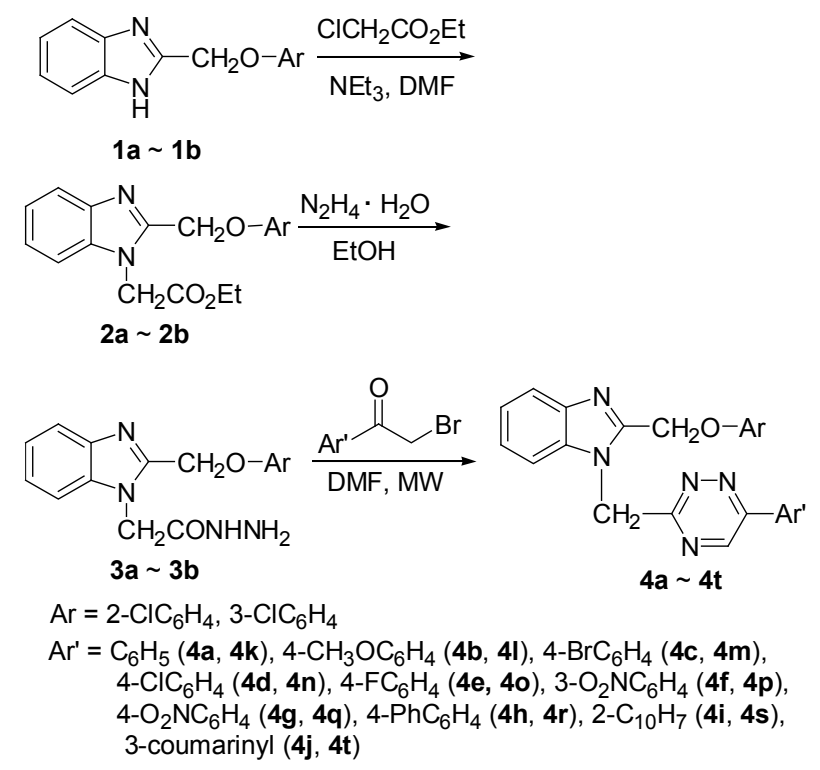

Scheme 1

为了探索出最佳合成条件，我们以 2-(3-氯苯氧基 甲基)- $1 H$-苯并咪唑-1-乙酰肼 $(\mathbf{3 b})(1 \mathrm{mmol})$ 与溴代苯乙 酮 $(0.5 \mathrm{mmol})$ 在 DMF 中的缩合合成目标化合物 $\mathbf{4 k}$ 为例, 考察了微波辐射时间、辐射功率、反应温度、溶剂用量 对产品收率的影响, 实验结果见表 1.

表 1 影响化合物 4k 产率的因素

Table 1 The effects of some factors on the yields of compound $4 \mathbf{k}$

\begin{tabular}{cccccc}
\hline Entry & $\begin{array}{c}\text { Time/ } \\
\min \end{array}$ & $\begin{array}{c}\text { Temperature/ } \\
{ }^{\circ} \mathrm{C}\end{array}$ & $\begin{array}{c}\text { Power/ } \\
\mathrm{W}\end{array}$ & $\begin{array}{c}\text { Sovent/ } \\
\mathrm{mL}\end{array}$ & $\begin{array}{c}\text { Yield/ } \\
\%\end{array}$ \\
\hline 1 & 40 & 90 & 700 & 1.0 & 40.7 \\
2 & 40 & 90 & 800 & 1.0 & 43.9 \\
3 & 40 & 90 & 850 & 1.0 & 44.0 \\
4 & 30 & 80 & 800 & 1.0 & 35.0 \\
5 & 30 & 90 & 800 & 1.0 & 35.2 \\
6 & 30 & 100 & 800 & 1.0 & 44.4 \\
7 & 30 & 110 & 800 & 1.0 & 51.4 \\
8 & 20 & 110 & 800 & 1.0 & 44.9 \\
9 & 40 & 110 & 800 & 1.0 & 43.9 \\
10 & 30 & 110 & 800 & 1.5 & 56.5 \\
11 & 30 & 110 & 800 & 2.0 & 32.7 \\
\hline
\end{tabular}

由表 1 数据可知，当微波辐射时间、反应温度和反 应溶剂(DMF)的量确定时(表 1, Entries 1 3), 目标化合 物 $4 \mathbf{k}$ 的产率随着辐射功率的增加而增加, 并在 $850 \mathrm{~W}$ 达到最大值(44.0\%), 但与 $800 \mathrm{~W}$ 时的产率 $(43.9 \%)$ 接近. 当微波辐射时间、辐射功率和溶剂的量固定时(表 1 , Entries 4 7), 结果表明, $4 \mathbf{k}$ 的产率随着反应温度的升 高而增加, 反应温度为 $110{ }^{\circ} \mathrm{C}$ 时产率比较合适(51.4\%). 
当辐射功率、反应温度和溶剂用量确定时(表 1, Entries 7 9), 最佳反应时间为 $30 \mathrm{~min}, 4 \mathbf{k}$ 的产率达到最大值 (51.4\%). 但当反应时间超过 $30 \mathrm{~min}$ 后, 产率略有下降. 在此实验中, 我们选择 $N, N$-二甲基甲酰胺(DMF)为溶 剂, 是因为它的沸点较高 (本反应温度要求为 $110{ }^{\circ} \mathrm{C}$ ), 可以更有效地吸收微波能量. 由于底物浓度对该反应有 一定影响, 所以还考察了溶剂的用量(表 1, Entries 7, $10 \sim 11)$, 结果表明, 当溶剂量为 $1.5 \mathrm{~mL}$ 时, 产率达到峰 值, 但溶剂超过此值, 产率较低, 这是由于降低了反应 物分子之间的有效碰撞. 此外, 在优化条件下, 我们还 考察了沸点更高、极性更大的非质子性溶剂二甲基亚砜 (DMSO)对产物产率的影响, 结果发现, 在 DMSO 中产 物的收率较低(15.4\%), 这说明, 溶剂的极性过大也不利 于此反应. 由表 1 的数据可以看出, 与其它条件相比较, 微波辐射功率对产物收率的影响不大(表 1, Entries 1 $3)$, 这是因为, 功率增大只是使达到反应温度的时间缩 短. 相比之下, 反应温度(表 1 , Entries 4 7) 和反应物浓 度(表 1, Entries 7,10 11) 对产物收率的影响较大. 另 外, 我们在相同的优化条件 $\left(1.5 \mathrm{~mL} \mathrm{DMF}, 110{ }^{\circ} \mathrm{C}, 30\right.$ $\min )$ 、而无微波辐射作用下，进行了此反应，结果没有 得到 1,2,4-三嗪产物, 这说明了微波条件对此反应的必 要性.

在此优化的反应条件 $\left(1.5 \mathrm{~mL} \mathrm{DMF}, 110{ }^{\circ} \mathrm{C}, 800 \mathrm{~W}\right.$, $30 \mathrm{~min})$ 下, 利用微波辅助合成了一系列含有苯并咪唑 单元的 3,6-二取代-1,2,4-三嗪衍生物, 即 3-[(2-芳氧甲 基)苯并咪唑-1-亚甲基]-6-芳基-1,2,4-三嗪(4a４t)，结 果见表 2. 显然, 这种方法不仅适用于溴代苯乙酮的苯 环上连有吸电子或供电子基团，对稠合芳环或稠杂环的 溴代甲基酮也适用, 而且与经典方法 ${ }^{[18]}$ 相比较产率较 高(表 2 中化合物 4m, 4n, 4q). 因此, 该方法对此类化合 物的合成具有广泛的适用性.

对所合成的新目标化合物 $4 \mathrm{a} \sim \mathbf{4 t}$ 进行了
Cdc25B/PTP1B 的抑制活性篮选，对照药物分别为正钒 酸钠和齐墩果酸. 初篮选择单浓度 $(5 \mu \mathrm{g} / \mathrm{mL})$ 条件下，对 样品的活性进行测试. 对于在一定条件下表现出抑制 率 $>50 \%$ 的样品, 再测试活性剂量依赖关系, 即 $\mathrm{IC}_{50}$ 值, 其测试结果见表 3. 由测试结果可以看出，目标化合物 $\mathbf{4 g}$ 和 $4 \mathbf{j}$ 对 $\mathrm{Cdc} 25 \mathrm{~B}$ 表现出良好的抑制活性. 目标化合物 $4 a, 4 h, 4 j, 4 r$ 和 $4 s$ 对 PTP1B 表现出良好的抑制活性, 其 中, 化合物 $4 \mathbf{j}$ 对 PTP1B 显示出最高的抑制活性 $\left[\mathrm{IC}_{50}=\right.$ $(0.59 \pm 0.04) \mu \mathrm{g} / \mathrm{L}]$, 并高于阳性对照药物齐墩果酸 $\left[\mathrm{IC}_{50}=(0.95 \pm 0.07) \mu \mathrm{g} / \mathrm{L}\right]$, 化合物 $\mathbf{4 h}$ 的抑制活性 $\left[\mathrm{IC}_{50}=\right.$ $(1.09 \pm 0.25) \mu \mathrm{g} / \mathrm{L}]$ 与齐墩果酸相近. 值得注意的是, 化 合物 4j 对 Cdc25B 和 PTP1B 均具有抑制活性.

\section{2 结论}

采用微波辐射辅助, 以酰肼和溴代苯乙酮为原料, 合成出了 20 个新的含苯并咪唑单元的 3,6-二取代-1,2,4三嗪衍生物 4. 探索了反应时间、温度、辐射功率、溶 剂用量对产率的影响, 得到了最佳合成条件. 与经典方 法相比较，该合成方法具有反应时间短、操作简便、底 物范围广、易纯化、产率较高等优点. 对所合成的目标 化合物进行了 $\mathrm{Cdc} 25 \mathrm{~B}$ 和 PTP1B 抑制活性的篎选. 实验 结果表明, 目标化合物 $\mathbf{4 g}$ 和 $\mathbf{4 j}$ 对 Cdc25B 表现出良好 的抑制活性，目标化合物 $4 \mathbf{a}, 4 \mathbf{h}, 4 \mathbf{j}, 4 \mathbf{r}$ 和 $4 \mathrm{~s}$ 对 PTP1B 表现出良好的抑制活性. 目标化合物 $\mathbf{4 j}$ 可作为用于治 疗癌症和糖尿病的潜在的 $\mathrm{Cdc} 25 \mathrm{~B}$ 和 PTP1B 抑制剂 $\left[\mathrm{IC}_{50}=(2.16 \pm 0.27)\right.$ 和 $\left.(0.59 \pm 0.04) \mu \mathrm{g} / \mathrm{mL}\right]$.

\section{3 实验部分}

\section{1 仪器与试剂}

仪器: X-5 显微熔点测定仪(温度计未经校正); 德国

表 2 微波辐射下含有苯并咪唑单元的 3,6-二取代-1,2,4-三嗪衍生物的合成 ${ }^{a}$

Table 2 Synthesis of 3,6-disubstituted-1,2,4-triazines containing benzimidazole moiety under MW

\begin{tabular}{|c|c|c|c|c|c|c|c|}
\hline Compd. & $\mathrm{Ar}$ & $A r^{\prime}$ & Yield ${ }^{b} / \%$ & Compd. & $\mathrm{Ar}$ & $\mathrm{Ar}^{\prime}$ & Yield $^{b} / \%$ \\
\hline $4 a$ & $2-\mathrm{ClC}_{6} \mathrm{H}_{4}$ & $\mathrm{C}_{6} \mathrm{H}_{5}$ & 55.1 & $4 k$ & $3-\mathrm{ClC}_{6} \mathrm{H}_{4}$ & $\mathrm{C}_{6} \mathrm{H}_{5}$ & 56.5 \\
\hline $4 b$ & $2-\mathrm{ClC}_{6} \mathrm{H}_{4}$ & 4- $\mathrm{CH}_{3} \mathrm{OC}_{6} \mathrm{H}_{4}$ & 46.3 & 41 & $3-\mathrm{ClC}_{6} \mathrm{H}_{4}$ & 4- $\mathrm{CH}_{3} \mathrm{OC}_{6} \mathrm{H}_{4}$ & 42.4 \\
\hline $4 c$ & $2-\mathrm{ClC}_{6} \mathrm{H}_{4}$ & $4-\mathrm{BrC}_{6} \mathrm{H}_{4}$ & 46.2 & $4 m$ & $3-\mathrm{ClC}_{6} \mathrm{H}_{4}$ & $4-\mathrm{BrC}_{6} \mathrm{H}_{4}$ & $49.8(40.7)^{c}$ \\
\hline $4 d$ & $2-\mathrm{ClC}_{6} \mathrm{H}_{4}$ & $4-\mathrm{ClC}_{6} \mathrm{H}_{4}$ & 43.3 & $4 n$ & $3-\mathrm{ClC}_{6} \mathrm{H}_{4}$ & 4- $\mathrm{ClC}_{6} \mathrm{H}_{4}$ & $48.9(36.9)^{c}$ \\
\hline $4 e$ & $2-\mathrm{ClC}_{6} \mathrm{H}_{4}$ & $4-\mathrm{FC}_{6} \mathrm{H}_{4}$ & 56.5 & 40 & $3-\mathrm{ClC}_{6} \mathrm{H}_{4}$ & $4-\mathrm{FC}_{6} \mathrm{H}_{4}$ & 54.7 \\
\hline $4 f$ & $2-\mathrm{ClC}_{6} \mathrm{H}_{4}$ & $3-\mathrm{O}_{2} \mathrm{NC}_{6} \mathrm{H}_{4}$ & 52.1 & $4 p$ & $3-\mathrm{ClC}_{6} \mathrm{H}_{4}$ & $3-\mathrm{O}_{2} \mathrm{NC}_{6} \mathrm{H}_{4}$ & 42.4 \\
\hline $4 g$ & $2-\mathrm{ClC}_{6} \mathrm{H}_{4}$ & $4-\mathrm{O}_{2} \mathrm{NC}_{6} \mathrm{H}_{4}$ & 49.4 & $4 q$ & $3-\mathrm{ClC}_{6} \mathrm{H}_{4}$ & $4-\mathrm{O}_{2} \mathrm{NC}_{6} \mathrm{H}_{4}$ & $48.3(21.3)^{c}$ \\
\hline $4 \mathrm{~h}$ & $2-\mathrm{ClC}_{6} \mathrm{H}_{4}$ & 4- $\mathrm{PhC}_{6} \mathrm{H}_{4}$ & 43.3 & $4 r$ & $3-\mathrm{ClC}_{6} \mathrm{H}_{4}$ & 4- $\mathrm{PhC}_{6} \mathrm{H}_{4}$ & 44.4 \\
\hline $4 i$ & $2-\mathrm{ClC}_{6} \mathrm{H}_{4}$ & $2-\mathrm{C}_{10} \mathrm{H}_{7}$ & 47.7 & $4 s$ & $3-\mathrm{ClC}_{6} \mathrm{H}_{4}$ & $2-\mathrm{C}_{10} \mathrm{H}_{7}$ & 52.3 \\
\hline $4 j$ & 2- $\mathrm{ClC}_{6} \mathrm{H}_{4}$ & & 33.2 & $4 t$ & $3-\mathrm{ClC}_{6} \mathrm{H}_{4}$ & & 32.6 \\
\hline
\end{tabular}

到应条件：酰肼 $\mathbf{3 b}$ 与取代的溴代苯乙酮缩合, 在 EtOH-DMF 中回流 $20 \mathrm{~h} ;{ }^{b}$ 分离纯化后的产率; ${ }^{c}$ 括号内的数据为采用经典法合成所得 ${ }^{[18]}$. 
表 3 化合物 4 对 Cdc25B 和 PTP1B 的抑制活性

Table 3 Inhibitory activity of compounds 4 against Cdc25B and PTP1B

\begin{tabular}{|c|c|c|c|c|}
\hline \multirow{2}{*}{ Compd. } & \multirow{2}{*}{$\mathrm{Ar}$} & \multirow{2}{*}{$\mathrm{Ar}^{\prime}$} & \multicolumn{2}{|c|}{$\mathrm{IC}_{50} /(\mu \mathrm{g} / \mathrm{L})^{a}$} \\
\hline & & & $\mathrm{Cdc} 25 \mathrm{~B}$ & PTP1B \\
\hline $4 a$ & $2-\mathrm{ClC}_{6} \mathrm{H}_{4}$ & $\mathrm{C}_{6} \mathrm{H}_{5}$ & $\mathrm{NA}^{b}$ & $1.64 \pm 0.46$ \\
\hline $4 b$ & $2-\mathrm{ClC}_{6} \mathrm{H}_{4}$ & $4-\mathrm{CH}_{3} \mathrm{OC}_{6} \mathrm{H}_{4}$ & NA & NA \\
\hline $4 c$ & $2-\mathrm{ClC}_{6} \mathrm{H}_{4}$ & $4-\mathrm{BrC}_{6} \mathrm{H}_{4}$ & NA & NA \\
\hline $4 d$ & $2-\mathrm{ClC}_{6} \mathrm{H}_{4}$ & 4- $\mathrm{ClC}_{6} \mathrm{H}_{4}$ & NA & NA \\
\hline $4 e$ & $2-\mathrm{ClC}_{6} \mathrm{H}_{4}$ & $4-\mathrm{FC}_{6} \mathrm{H}_{4}$ & NA & NA \\
\hline $4 f$ & $2-\mathrm{ClC}_{6} \mathrm{H}_{4}$ & $3-\mathrm{O}_{2} \mathrm{NC}_{6} \mathrm{H}_{4}$ & NA & NA \\
\hline $4 g$ & $2-\mathrm{ClC}_{6} \mathrm{H}_{4}$ & $4-\mathrm{O}_{2} \mathrm{NC}_{6} \mathrm{H}_{4}$ & $8.86 \pm 0.52$ & NA \\
\hline $4 h$ & $2-\mathrm{ClC}_{6} \mathrm{H}_{4}$ & 4- $\mathrm{PhC}_{6} \mathrm{H}_{4}$ & NA & $1.09 \pm 0.25$ \\
\hline $4 i$ & $2-\mathrm{ClC}_{6} \mathrm{H}_{4}$ & $2-\mathrm{C}_{10} \mathrm{H}_{7}$ & NA & NA \\
\hline $4 j$ & $2-\mathrm{ClC}_{6} \mathrm{H}_{4}$ & & $2.16 \pm 0.27$ & $0.59 \pm 0.04$ \\
\hline $4 k$ & $3-\mathrm{ClC}_{6} \mathrm{H}_{4}$ & $\mathrm{C}_{6} \mathrm{H}_{5}$ & NA & NA \\
\hline 41 & $3-\mathrm{ClC}_{6} \mathrm{H}_{4}$ & $4-\mathrm{CH}_{3} \mathrm{OC}_{6} \mathrm{H}_{4}$ & NA & NA \\
\hline $4 m$ & $3-\mathrm{ClC}_{6} \mathrm{H}_{4}$ & $4-\mathrm{BrC}_{6} \mathrm{H}_{4}$ & NA & NA \\
\hline $4 n$ & $3-\mathrm{ClC}_{6} \mathrm{H}_{4}$ & $4-\mathrm{ClC}_{6} \mathrm{H}_{4}$ & NA & NA \\
\hline 40 & $3-\mathrm{ClC}_{6} \mathrm{H}_{4}$ & $4-\mathrm{FC}_{6} \mathrm{H}_{4}$ & NA & NA \\
\hline $4 p$ & $3-\mathrm{ClC}_{6} \mathrm{H}_{4}$ & $3-\mathrm{O}_{2} \mathrm{NC}_{6} \mathrm{H}_{4}$ & NA & NA \\
\hline $4 q$ & $3-\mathrm{ClC}_{6} \mathrm{H}_{4}$ & $4-\mathrm{O}_{2} \mathrm{NC}_{6} \mathrm{H}_{4}$ & NA & NA \\
\hline $4 r$ & $3-\mathrm{ClC}_{6} \mathrm{H}_{4}$ & 4- $\mathrm{PhC}_{6} \mathrm{H}_{4}$ & NA & $4.35 \pm 0.18$ \\
\hline $4 s$ & $3-\mathrm{ClC}_{6} \mathrm{H}_{4}$ & $2-\mathrm{C}_{10} \mathrm{H}_{7}$ & NA & $1.83 \pm 0.23$ \\
\hline $4 t$ & $3-\mathrm{ClC}_{6} \mathrm{H}_{4}$ & & NA & NA \\
\hline $\mathrm{Na}_{3} \mathrm{VO}_{4}$ & - & - & $1.42 \pm 0.08$ & - \\
\hline Oleanolic acid & - & - & - & $0.95 \pm 0.07$ \\
\hline
\end{tabular}

${ }^{a}$ 目标化合物的测试浓度为 $5 \mu \mathrm{g} / \mathrm{mL}$, 均溶于 DMSO 中. ${ }^{b} \mathrm{NA}$ 表示没有活性(抑制率 $<50 \%$ ).

TENSOR 27 型 FT-IR 红外光谱仪, WGH-30 型双光束红 外分光光度计, KBr 压片; 美国 Varian INOVA $400 \mathrm{MHz}$ 核磁共振仪, DMSO- $d_{6} / \mathrm{CDCl}_{3}$ 为溶剂, TMS 为内标; 德 国 Elementar 公司 Vario EL 型元素分析仪; 北京祥鹄科 技发展有限公司 XH-100B 型电脑微波催化合成/萃取仪; 所用试剂均为分析纯.

\section{2 合成}

\subsection{1 中间体的合成}

2-芳氧甲基苯并咪唑(1)、2-芳氧甲基苯并咪唑-1乙酸乙酯(2)和 2-芳氧甲基苯并咪唑-1-乙酰肼(3)按照参 考文献[10]合成. 其中化合物 $\mathbf{2 b}$ 和 $\mathbf{3 b}$ 为未知物.

化合物 $\mathbf{2 b}$ : 得 $2.77 \mathrm{~g}$, 白色针状晶体, 产率 $68.0 \%$. m.p. $133.3 \sim 134.2{ }^{\circ} \mathrm{C} ;{ }^{1} \mathrm{H}$ NMR (500 MHz, DMSO- $\left.d_{6}\right) \delta$ : 7.68 (d, $\left.J=7.7 \mathrm{~Hz}, 1 \mathrm{H}, \mathrm{BZM}-\mathrm{H}_{4}\right), 7.58$ (d, $J=7.9 \mathrm{~Hz}, 1 \mathrm{H}$, BZM-H-7), 7.33 (t, $J=8.2 \mathrm{~Hz}, 1 \mathrm{H}, \mathrm{ArH}), 7.29$ (td, $J=7.6$, $1.1 \mathrm{~Hz}, 1 \mathrm{H}$, BZM-H-6), 7.24 (td, $J=7.5,1.0 \mathrm{~Hz}, 1 \mathrm{H}$, BZM-H-5), 7.14 (dd, $J=2.2,2.1 \mathrm{~Hz}, 1 \mathrm{H}, \mathrm{ArH}), 7.04$ (dd, $J=8.0,1.4 \mathrm{~Hz}, 1 \mathrm{H}, \mathrm{ArH}), 7.01(\mathrm{dd}, J=8.4,2.3 \mathrm{~Hz}, 1 \mathrm{H}$, ArH), 5.44 (s, 2H, $\left.\mathrm{CH}_{2} \mathrm{O}\right), 5.28\left(\mathrm{~s}, 2 \mathrm{H}, \mathrm{NCH}_{2}\right), 4.11$ (q, $\left.J=7.1 \mathrm{~Hz}, 2 \mathrm{H}, \mathrm{CO}_{2} \mathrm{CH}_{2}\right), 1.15\left(\mathrm{t}, J=7.1 \mathrm{~Hz}, 3 \mathrm{H}, \mathrm{CH}_{3}\right)$; IR
(KBr) v: 3059, 2987, 2868, 2860, 1743, 1625, 1595, 1475, 1434, 1380, 1291, 1231, $1023 \mathrm{~cm}^{-1}$. Anal. calcd for $\mathrm{C}_{18} \mathrm{H}_{17} \mathrm{ClN}_{2} \mathrm{O}_{3}$ : C 62.70, H 4.97, N 8.12; found $\mathrm{C} 62.51, \mathrm{H}$ 5.11, N 8.01.

化合物 $3 \mathrm{~b}$ : 得 $3.4 \mathrm{~g}$, 白色针状晶体 $3 \mathrm{~b}$, 产率 $88.6 \%$. m.p. $230.3 \sim 231.3{ }^{\circ} \mathrm{C} ;{ }^{1} \mathrm{H}$ NMR $\left(500 \mathrm{MHz}, \mathrm{DMSO}-d_{6}\right) \delta$ : 9.48 (s, 1H, CONH), 7.65 (d, $J=7.9 \mathrm{~Hz}, 1 \mathrm{H}, \mathrm{BZM}-\mathrm{H}-4)$, 7.52 (d, $J=8.0 \mathrm{~Hz}, 1 \mathrm{H}, \mathrm{BZM}-\mathrm{H}-7), 7.32$ (t, $J=8.2 \mathrm{~Hz}, 1 \mathrm{H}$, ArH), 7.28 (td, $J=7.6,1.0 \mathrm{~Hz}, 1 \mathrm{H}, \mathrm{BZM}-\mathrm{H}-6), 7.18 \sim 7.25$ (m, 2H, BZM-H $\left.{ }_{5}, \mathrm{ArH}\right), 7.07$ (ddd, $J=8.4,2.3,0.6 \mathrm{~Hz}$, 1H, ArH), 7.03 (ddd, $J=7.9,1.9,0.6 \mathrm{~Hz}, 1 \mathrm{H}, \mathrm{ArH}), 5.43$ (s, 2H, $\left.\mathrm{CH}_{2} \mathrm{O}\right), 4.94$ (s, 2H, $\left.\mathrm{NCH}_{2}\right), 4.31\left(\mathrm{~s}, 2 \mathrm{H}, \mathrm{NH}_{2}\right)$; IR (KBr) v: 3296, 3052, 2946, 2862, 1660, 1595, 1535, 1475, 1434, 1375, 1284, 1238, $1029 \mathrm{~cm}^{-1}$. Anal. calcd for $\mathrm{C}_{16} \mathrm{H}_{15} \mathrm{ClN}_{4} \mathrm{O}_{2}$ : C 58.10, $\mathrm{H}$ 4.57, N 16.94; found $\mathrm{C} 58.33$, $\mathrm{H} 4.78, \mathrm{~N} 16.77$.

\subsection{2 目标化合物 $\mathbf{4 a} \sim \mathbf{4 t}$ 的合成通法}

在干燥的 $50 \mathrm{~mL}$ 三颈瓶中加入 $1 \mathrm{mmol} 2$-芳氧甲基 苯并咪坐-1-乙酰肼 $(3) 、 0.5 \mathrm{mmol}$ 溴代苯乙酮和 $1.5 \mathrm{~mL}$ $\mathrm{DMF}$ ，微波辐射 $30 \mathrm{~min}$, 反应结束后，将反应瓶冷却至 
室温, 加入少量冷水, 用饱和碳酸钠溶液调节溶液 $\mathrm{pH}=7$, 过滤、用水洗涤滤饼, 固体粗产品用 $\mathrm{DMF} /$ DMF- $\mathrm{H}_{2} \mathrm{O}$ 重结晶, 即得到目标化合物纯品.

3-[2-(2-氯苯氧甲基)苯并咪唑-1-亚甲基]-6-苯基1,2,4-三嗪(4a): 浅黄色固体, 收率 55.1\%. m.p. 202.4 $203.2{ }^{\circ} \mathrm{C} ;{ }^{1} \mathrm{H}$ NMR (400 MHz, DMSO- $\left.d_{6}\right) \delta: 5.51(\mathrm{~s}, 2 \mathrm{H}$, $\left.\mathrm{CH}_{2} \mathrm{O}\right), 6.09$ (s, 2H, $\left.\mathrm{NCH}_{2}\right), 6.78(\mathrm{~d}, J=8.4 \mathrm{~Hz}, 1 \mathrm{H}, \mathrm{ArH})$, 6.85 (brs, $1 \mathrm{H}, \mathrm{ArH}), 6.94$ (d, $J=8.0 \mathrm{~Hz}, 1 \mathrm{H}, \mathrm{ArH}), 7.18$ (t, $J=8.0 \mathrm{~Hz}, 1 \mathrm{H}, \mathrm{ArH}$ ), $7.23 \sim 7.33$ (m, 2H, BZM-H-5, H-6), $7.55 \sim 7.63\left(\mathrm{~m}, 3 \mathrm{H}, \mathrm{Ar}^{\prime} \mathrm{H}\right), 7.66$ (d, $J=7.2 \mathrm{~Hz}, 1 \mathrm{H}, \mathrm{BZM}-$ H-7), 7.72 (d, $J=7.2 \mathrm{~Hz}, 1 \mathrm{H}$, BZM-H-4), 8.13 (brs, 2H, Ar'H), 9.27 (s, 1H, triazine-H); IR (KBr) v: 3060, 3036, 2928, 2860, 1624, 1596, 1582, 1480, 1462, 1284, 1232, $1030 \mathrm{~cm}^{-1}$. Anal. calcd for $\mathrm{C}_{24} \mathrm{H}_{18} \mathrm{ClN}_{5} \mathrm{O}: \mathrm{C} 67.37, \mathrm{H} 4.24$, N 16.37; found C 67.16, H 4.42, N 16.48.

3-[2-(2-氯苯氧甲基)苯并咪唑-1-亚甲基]-6-(4-甲氧 基苯基)-1,2,4-三嗪(4b): 淡黄色固体, 收率 46.3\%. m.p. $182.3 \sim 183.1{ }^{\circ} \mathrm{C} ;{ }^{1} \mathrm{H}$ NMR (400 MHz, DMSO- $d_{6}$ ) $\delta: 3.84$ (s, $\left.3 \mathrm{H}, \mathrm{CH}_{3} \mathrm{O}\right), 5.58\left(\mathrm{~s}, 2 \mathrm{H}, \mathrm{CH}_{2} \mathrm{O}\right), 6.10\left(\mathrm{~s}, 2 \mathrm{H}, \mathrm{NCH}_{2}\right)$, $6.92(\mathrm{t}, J=7.4 \mathrm{~Hz}, 1 \mathrm{H}, \mathrm{ArH}), 7.13(\mathrm{~d}, J=8.8 \mathrm{~Hz}, 2 \mathrm{H}$, Ar'H), $7.20 \sim 7.35$ (m, 4H, BZM-H-5, H-6, ArH $\times 2$ ), 7.40 (d, $J=7.6 \mathrm{~Hz}, 1 \mathrm{H}, \mathrm{ArH}), 7.63(\mathrm{~d}, J=8.8 \mathrm{~Hz}, 1 \mathrm{H}, \mathrm{BZM}-$ H-7), 7.73 (d, $J=7.2 \mathrm{~Hz}, 1 \mathrm{H}$, BZM-H-4), 8.08 (d, $J=8.8$ $\left.\mathrm{Hz}, 2 \mathrm{H}, \mathrm{Ar}^{\prime} \mathrm{H}\right), 9.19$ (s, 1H, triazine-H); IR (KBr) v: 3058, 3046, 2937, 2839, 1607, 1471, 1244, $1022 \mathrm{~cm}^{-1}$. Anal. calcd for $\mathrm{C}_{25} \mathrm{H}_{20} \mathrm{ClN}_{5} \mathrm{O}_{2}$ : C 65.57, H 4.40, N 15.29; found C 65.69, H 4.29, N 15.45 .

3-[2-(2-氯苯氧甲基)苯并咪唑-1-亚甲基]-6-(4-溴苯 基)-1,2,4-三嗪(4c): 淡黄色粉末, 收率 46.2\%. m.p. $210.2 \sim 211.1{ }^{\circ} \mathrm{C} ;{ }^{1} \mathrm{H}$ NMR (400 MHz, DMSO- $d_{6}$ ) $\delta: 5.58$ (s, $2 \mathrm{H}, \mathrm{CH}_{2} \mathrm{O}$ ), 6.14 (s, $\left.2 \mathrm{H}, \mathrm{NCH}_{2}\right), 6.91(\mathrm{t}, J=8.4 \mathrm{~Hz}, 1 \mathrm{H}$, ArH), $7.20 \sim 7.35$ (m, 4H, BZM-H-5, H-6, ArH $\times 2), 7.39$ (d, $J=8.4 \mathrm{~Hz}, 1 \mathrm{H}, \mathrm{ArH}), 7.64(\mathrm{~d}, J=7.2 \mathrm{~Hz}, 1 \mathrm{H}, \mathrm{BZM}-$ H-7), 7.73 (d, $J=7.2 \mathrm{~Hz}, 1 \mathrm{H}$, BZM-H-4), 7.80 (d, $J=8.4$ Hz, 2H, Ar'H), 8.05 (d, J=8.4 Hz, 2H, Ar'H), 9.25 (s, 1H, triazine-H); IR (KBr) v: 3064, 2932, 2860, 1620, 1592, $1488,1464,1238,1058 \mathrm{~cm}^{-1}$. Anal. calcd for $\mathrm{C}_{24} \mathrm{H}_{17} \mathrm{Br}-$ $\mathrm{ClN}_{5} \mathrm{O}$ : C 56.88, H 3.38, N 13.82; found C 56.95, H 3.50, $\mathrm{N} 13.68$.

3-[2-(2-氯苯氧甲基)苯并咪唑-1-亚甲基]-6-(4-氯苯 基)-1,2,4-三嗪(4d): 浅黄色固体, 收率 43.3\%. m.p. 199.2 199.7 ${ }^{\circ} \mathrm{C} ;{ }^{1} \mathrm{H}$ NMR (400 MHz, DMSO- $d_{6}$ ) $\delta: 5.58$ $\left(\mathrm{s}, 2 \mathrm{H}, \mathrm{CH}_{2} \mathrm{O}\right), 6.15\left(\mathrm{~s}, 2 \mathrm{H}, \mathrm{NCH}_{2}\right), 6.92(\mathrm{t}, J=7.4 \mathrm{~Hz}, 1 \mathrm{H}$, ArH), $7.20 \sim 7.35$ (m, 4H, BZM-H-5, H-6, ArH $\times 2), 7.39$ (d, $J=7.6 \mathrm{~Hz}, 1 \mathrm{H}, \mathrm{ArH}), 7.60 \sim 7.70$ (m, 3H, BZM-H-7,
Ar'H $\times 2), 7.73$ (d, $J=8.8 \mathrm{~Hz}, 1 \mathrm{H}, \mathrm{BZM}-\mathrm{H}-4), 8.13$ (d, $J=$ $8.8 \mathrm{~Hz}, 2 \mathrm{H}, \mathrm{Ar} \mathrm{H}), 9.25$ (s, 1H, triazine-H); IR (KBr) v: 3059, 2961, 2853, 1618, 1591, 1488, 1461, 1238, 1060 $\mathrm{cm}^{-1}$. Anal. calcd for $\mathrm{C}_{24} \mathrm{H}_{17} \mathrm{Cl}_{2} \mathrm{~N}_{5} \mathrm{O}: \mathrm{C} 62.35, \mathrm{H} 3.71, \mathrm{~N}$ 15.15; found C 62.17, H 3.63, N 15.35.

3-[2-(2-氯苯氧甲基)苯并咪唑-1-亚甲基]-6-(4-氟苯 基)-1,2,4-三嗪(4e)：浅黄色晶体，收率 56.5\%. m.p. $167.9 \sim 168.8{ }^{\circ} \mathrm{C} ;{ }^{1} \mathrm{H}$ NMR (400 MHz, DMSO- $d_{6}$ ) $\delta: 5.58$ (s, $\left.2 \mathrm{H}, \mathrm{CH}_{2} \mathrm{O}\right), 6.14\left(\mathrm{~s}, 2 \mathrm{H}, \mathrm{NCH}_{2}\right), 6.92(\mathrm{t}, J=7.6 \mathrm{~Hz}, 1 \mathrm{H}$, ArH), $7.20 \sim 7.33(\mathrm{~m}, 4 \mathrm{H}, \mathrm{BZM}-\mathrm{H}-5, \mathrm{H}-6, \mathrm{ArH} \times 2)$, $7.37 \sim 7.47\left(\mathrm{~m}, 3 \mathrm{H}, \mathrm{ArH}, \operatorname{Ar}^{\prime} \mathrm{H} \times 2\right), 7.64(\mathrm{~d}, J=8.4 \mathrm{~Hz}$, 1H, BZM-H-7), 7.73 (d, $J=7.2 \mathrm{~Hz}, 1 \mathrm{H}, \mathrm{BZM}-\mathrm{H}-4), 8.17$ (dd, $\left.J=8.6 \mathrm{~Hz}, 5.8 \mathrm{~Hz}, 2 \mathrm{H}, \mathrm{Ar}^{\prime} \mathrm{H}\right), 9.24$ (s, $1 \mathrm{H}$, triazine-H); IR (KBr) v: 3064, 2939, 2853, 1629, 1602, 1488, 1466, $1238,1060 \mathrm{~cm}^{-1}$. Anal. calcd for $\mathrm{C}_{24} \mathrm{H}_{17} \mathrm{ClFN}_{5} \mathrm{O}: \mathrm{C} 64.65$, H 3.84, N 15.71; found C 64.50, H 3.68, N 15.61 .

3-[2-(2-氯苯氧甲基)苯并咪唑-1-亚甲基]-6-(3-硝基 苯基)-1,2,4-三嗪(4f): 米黄色固体, 收率 52.1\%. m.p. $188.6 \sim 189.5{ }^{\circ} \mathrm{C} ;{ }^{1} \mathrm{H}$ NMR (400 MHz, DMSO- $d_{6}$ ) $\delta: 5.59$ (s, $\left.2 \mathrm{H}, \mathrm{CH}_{2} \mathrm{O}\right), 6.19\left(\mathrm{~s}, 2 \mathrm{H}, \mathrm{NCH}_{2}\right), 6.92(\mathrm{t}, J=7.8 \mathrm{~Hz}, 1 \mathrm{H}$, ArH), 7.23 (d, $J=8.0 \mathrm{~Hz}, 1 \mathrm{H}, \mathrm{ArH}), 7.25 \sim 7.33(\mathrm{~m}, 3 \mathrm{H}$, BZM-H-5, H-6, ArH), 7.40 (d, $J=8.4 \mathrm{~Hz}, 1 \mathrm{H}, \mathrm{ArH}), 7.65$ (d, $J=6.8 \mathrm{~Hz}, 1 \mathrm{H}$, BZM-H-7), $7.74(\mathrm{~d}, J=8.8 \mathrm{~Hz}, 1 \mathrm{H}$, BZM-H-4), 7.90 (t, $J=8.0 \mathrm{~Hz}, 1 \mathrm{H}, \mathrm{Ar}^{\prime} \mathrm{H}$ ), 8.43 (d, $J=7.2$ $\left.\mathrm{Hz}, 1 \mathrm{H}, \mathrm{Ar}^{\prime} \mathrm{H}\right), 8.54$ (d, J=7.6 Hz, 1H, Ar'H), 8.89 (s, 1H, Ar'H), 9.40 (s, 1H, triazine-H); IR (KBr) v: 3076, 2936, 2866, 1620, 1590, 1530, 1484, 1466, 1352, 1278, 1244, $1058 \mathrm{~cm}^{-1}$. Anal. calcd for $\mathrm{C}_{24} \mathrm{H}_{17} \mathrm{ClN}_{6} \mathrm{O}_{3}: \mathrm{C} 60.96, \mathrm{H}$ 3.62, N 17.77; found C 60.71, H 3.79, N 17.82 .

3-[2-(2-氯苯氧甲基)苯并咪唑-1-亚甲基]-6-(4-硝基 苯基)-1,2,4-三嗪(4g)：黄绿色固体，收率 49.4\%. m.p. $225.7 \sim 226.8{ }^{\circ} \mathrm{C} ;{ }^{1} \mathrm{H}$ NMR (400 MHz, DMSO- $d_{6}$ ) $\delta: 5.59$ $\left(\mathrm{s}, 2 \mathrm{H}, \mathrm{CH}_{2} \mathrm{O}\right), 6.20\left(\mathrm{~s}, 2 \mathrm{H}, \mathrm{NCH}_{2}\right), 6.91(\mathrm{t}, J=7.2 \mathrm{~Hz}, 1 \mathrm{H}$, ArH), 7.23 (d, $J=8.4 \mathrm{~Hz}, 1 \mathrm{H}, \mathrm{ArH}), 7.25 \sim 7.35(\mathrm{~m}, 3 \mathrm{H}$, BZM-H-5, H-6, ArH), 7.40 (d, J=8.4 Hz, 1H, ArH), 7.65 (d, $J=6.8 \mathrm{~Hz}, 1 \mathrm{H}$, BZM-H-7), $7.74(\mathrm{~d}, J=8.8 \mathrm{~Hz}, 1 \mathrm{H}$, BZM-H-4), 8.37 (d, $J=9.2 \mathrm{~Hz}, 2 \mathrm{H}, \mathrm{Ar}^{\prime} \mathrm{H}$ ), 8.40 (d, $J=8.8$ $\mathrm{Hz}, 2 \mathrm{H}, \mathrm{Ar} \mathrm{H}), 9.36$ (s, $1 \mathrm{H}$, triazine-H); IR (KBr) $v: 3076$, 2932, 2860, 1606, 1592, 1524, 1486, 1464, 1352, 1242, $1054 \mathrm{~cm}^{-1}$. Anal. calcd for $\mathrm{C}_{24} \mathrm{H}_{17} \mathrm{ClN}_{6} \mathrm{O}_{3}: \mathrm{C} 60.96, \mathrm{H}$ 3.62, N 17.77; found C 61.19, H 3.71, N 17.62.

3-[2-(2-氯苯氧甲基)苯并咪唑-1-亚甲基]-6-联苯基1,2,4-三嗪(4h): 浅黄色粉末, 收率 43.3\%. m.p. 203.3 $204.4{ }^{\circ} \mathrm{C} ;{ }^{1} \mathrm{H}$ NMR (400 MHz, DMSO- $d_{6}$ ) $\delta: 5.60$ (s, $2 \mathrm{H}$, $\left.\mathrm{CH}_{2} \mathrm{O}\right), 6.15$ (s, $\left.2 \mathrm{H}, \mathrm{NCH}_{2}\right), 6.92(\mathrm{t}, J=7.6 \mathrm{~Hz}, 1 \mathrm{H}, \mathrm{ArH})$, 
$7.20 \sim 7.35$ (m, 4H, BZM-H-5, H-6, ArH $\times 2), 7.37 \sim 7.47$ (m, 2H, ArH, Ar'H), 7.51 (t, J=7.6 Hz, 2H, Ar'H), 7.66 (d, $J=8.0 \mathrm{~Hz}, 1 \mathrm{H}$, BZM-H-7), 7.74 (d, $J=6.8 \mathrm{~Hz}, 1 \mathrm{H}$, BZM-H-4), 7.78 (d, J=7.6 Hz, 2H, Ar'H), 7.90 (d, $J=8.4$ $\mathrm{Hz}, 2 \mathrm{H}, \mathrm{Ar}^{\prime} \mathrm{H}$ ), 8.21 (d, J=8.4 Hz, 2H, Ar'H), 9.30 (s, 1H, triazine-H); IR (KBr) v: 3060, 2928, 2857, 1610, 1592, $1486,1466,1242,1060 \mathrm{~cm}^{-1}$. Anal. calcd for $\mathrm{C}_{30} \mathrm{H}_{22} \mathrm{Cl}-$ $\mathrm{N}_{5} \mathrm{O}$ : C 71.49, H 4.40, N 13.90; found C 71.62, H 4.26, N 13.72 .

3-[2-(2-氯苯氧甲基)苯并咪唑-1-亚甲基]-6-(2-荟 基)-1,2,4-三嗪(4i)：淡黄色晶体，收率 47.7\%. m.p. $192.5 \sim 193.4{ }^{\circ} \mathrm{C} ;{ }^{1} \mathrm{H}$ NMR (400 MHz, DMSO- $d_{6}$ ) $\delta: 5.60$ (s, 2H, $\left.\mathrm{CH}_{2} \mathrm{O}\right), 6.17$ (s, 2H, $\left.\mathrm{NCH}_{2}\right), 6.92$ (t, $J=7.4 \mathrm{~Hz}, 1 \mathrm{H}$, ArH), 7.24 (d, J=8.0 Hz, 1H, ArH), $7.25 \sim 7.35$ (m, 3H, BZM-H-5, H-6, ArH), 7.41 (d, $J=8.0 \mathrm{~Hz}, 1 \mathrm{H}, \mathrm{ArH})$, $7.55 \sim 7.65\left(\mathrm{~m}, 2 \mathrm{H}, \mathrm{Ar}^{\prime} \mathrm{H}\right), 7.66(\mathrm{~d}, J=6.8 \mathrm{~Hz}, 1 \mathrm{H}$, BZM-H-7), 7.74 (d, $J=8.4 \mathrm{~Hz}, 1 \mathrm{H}, \mathrm{BZM-H}-4), 8.01$ (d, $\left.J=6.8 \mathrm{~Hz}, 1 \mathrm{H}, \mathrm{Ar}^{\prime} \mathrm{H}\right), 8.07$ (d, $\left.J=6.8 \mathrm{~Hz}, 1 \mathrm{H}, \mathrm{Ar}^{\prime} \mathrm{H}\right), 8.12$ (d, $\left.J=8.4 \mathrm{~Hz}, 1 \mathrm{H}, \mathrm{Ar}^{\prime} \mathrm{H}\right), 8.23$ (d, $\left.J=8.8 \mathrm{~Hz}, 1 \mathrm{H}, \mathrm{Ar}^{\prime} \mathrm{H}\right)$, 8.73 (s, 1H, Ar'H), 9.40 (s, 1H, triazine-H); IR (KBr) v: 3058, 2939, 2858, 1618, 1585, 1477, 1244, $1027 \mathrm{~cm}^{-1}$. Anal. calcd for $\mathrm{C}_{28} \mathrm{H}_{20} \mathrm{ClN}_{5} \mathrm{O}: \mathrm{C} 70.36, \mathrm{H} 4.22$, N 14.65; found $\mathrm{C} 70.26, \mathrm{H} 4.37, \mathrm{~N} 14.88$.

3-[2-(2-氯苯氧甲基)苯并咪唑-1-亚甲基]-6-香豆素 基-1,2,4-三嗪(4j): 褐色固体, 收率 33.2\%. m.p. 178.6 $179.5{ }^{\circ} \mathrm{C} ;{ }^{1} \mathrm{H}$ NMR (400 MHz, DMSO- $\left.d_{6}\right) \delta: 5.59$ (s, 2H, $\left.\mathrm{CH}_{2} \mathrm{O}\right), 6.19$ (s, 2H, $\left.\mathrm{NCH}_{2}\right), 6.92$ (t, J=7.6 Hz, 1H, ArH), $7.23(\mathrm{~d}, J=8.0 \mathrm{~Hz}, 1 \mathrm{H}, \mathrm{ArH}), 7.25 \sim 7.35(\mathrm{~m}, 4 \mathrm{H}$, BZM-H-5, H-6, ArH, Ar'H), 7.40 (d, $J=8.0 \mathrm{~Hz}, 1 \mathrm{H}, \mathrm{ArH})$, 7.65 (d, $J=6.8 \mathrm{~Hz}, 1 \mathrm{H}$, BZM-H-7), 7.74 (d, $J=6.8 \mathrm{~Hz}$, 1H, BZM-H-4), 7.90 (t, $\left.J=7.8 \mathrm{~Hz}, 1 \mathrm{H}, \mathrm{Ar}^{\prime} \mathrm{H}\right), 8.43$ (d, $J=$ $\left.6.8 \mathrm{~Hz}, 1 \mathrm{H}, \mathrm{Ar}^{\prime} \mathrm{H}\right), 8.54$ (d, J=7.6 Hz, 1H, Ar'H), 8.89 (s, 1H, Ar'H), 9.40 (s, 1H, triazine-H); IR (KBr) v: 3068, 2948, 2862, 1718, 1610, 1590, 1486, 1464, 1278, 1248, $1060 \mathrm{~cm}^{-1}$. Anal. calcd for $\mathrm{C}_{27} \mathrm{H}_{18} \mathrm{ClN}_{5} \mathrm{O}_{3}: \mathrm{C} 65.39, \mathrm{H}$ 3.66, N 14.12; found C 65.28, H 3.84, N 14.25.

3-[2-(3-氯苯氧甲基)苯并咪唑-1-亚甲基]-6-苯基1,2,4-三嗪(4k): 淡黄色粉末，收率 56.5\%. m.p. 196.6 $197.5{ }^{\circ} \mathrm{C} ;{ }^{1} \mathrm{H}$ NMR (400 MHz, $\left.\mathrm{CDCl}_{3}\right) \delta$ : 5.67 (s, 2H, $\left.\mathrm{CH}_{2} \mathrm{O}\right), 5.99\left(\mathrm{~s}, 2 \mathrm{H}, \mathrm{NCH}_{2}\right), 6.90 \sim 7.05(\mathrm{~m}, 3 \mathrm{H}, \mathrm{ArH})$, $7.17(\mathrm{t}, J=8.0 \mathrm{~Hz}, 1 \mathrm{H}, \operatorname{ArH}), 7.30 \sim 7.43(\mathrm{~m}, 2 \mathrm{H}$, BZM-H-5, H-6), $7.47 \sim 7.65$ (m, 4H, BZM-H-7, Ar'H×3), $7.80 \sim 7.90$ (m, 1H, BZM-H-4), $7.95 \sim 8.10$ (m, 2H, Ar'H), $8.84(\mathrm{~s}, 1 \mathrm{H}$, triazine-H); IR $(\mathrm{KBr}) v: 3060,2932,2868$, $1618,1596,1588,1464,1230,1030 \mathrm{~cm}^{-1}$. Anal. calcd for
$\mathrm{C}_{24} \mathrm{H}_{18} \mathrm{ClN}_{5} \mathrm{O}$ : C 67.37, H 4.24, N 16.37; found $\mathrm{C} 67.15, \mathrm{H}$ 4.36, N 16.22.

3-[2-(3-氯苯氧甲基)苯并咪唑-1-亚甲基]-6-(4-甲氧 基苯基)-1,2,4-三嗪(4l)：淡黄色固体，收率 42.4\%. m.p. $185.6 \sim 186.2{ }^{\circ} \mathrm{C} ;{ }^{1} \mathrm{H}$ NMR (400 MHz, DMSO- $d_{6}$ ) $\delta: 3.84$ $\left(\mathrm{s}, 3 \mathrm{H}, \mathrm{CH}_{3} \mathrm{O}\right), 5.50\left(\mathrm{~s}, 2 \mathrm{H}, \mathrm{CH}_{2} \mathrm{O}\right), 6.04\left(\mathrm{~s}, 2 \mathrm{H}, \mathrm{NCH}_{2}\right)$, 6.78 (d, $J=10.0 \mathrm{~Hz}, 1 \mathrm{H}, \operatorname{ArH}), 6.86$ (s, 1H, ArH), 6.94 (d, $J=8.4 \mathrm{~Hz}, 1 \mathrm{H}, \mathrm{ArH}), 7.13$ (d, $\left.J=8.4 \mathrm{~Hz}, 2 \mathrm{H}, \mathrm{Ar}^{\prime} \mathrm{H}\right), 7.18$ $(\mathrm{t}, J=8.0 \mathrm{~Hz}, 1 \mathrm{H}, \mathrm{ArH}), 7.20 \sim 7.33$ (m, 2H, BZM-H-5, H-6), 7.64 (d, $J=7.6 \mathrm{~Hz}, 1 \mathrm{H}, \mathrm{BZM}-\mathrm{H}-7), 7.71$ (d, $J=7.2$ Hz, 1H, BZM-H-4), 8.10 (d, J=8.4 Hz, 2H, Ar'H), 9.21 (s, $1 \mathrm{H}$, triazine-H); IR (KBr) v: 3053, 3031, 2934, 2836, 1607, 1591, 1477, 1461, 1260, $1033 \mathrm{~cm}^{-1}$. Anal. calcd for $\mathrm{C}_{25} \mathrm{H}_{20} \mathrm{ClN}_{5} \mathrm{O}_{2}$ : C 65.57, $\mathrm{H} 4.40, \mathrm{~N} 15.29$; found $\mathrm{C}$ 65.62, $\mathrm{H} 4.30, \mathrm{~N} 15.17$.

3-[2-(3-氯苯氧甲基)苯并咪唑-1-亚甲基]-6-(4-溴苯 基)-1,2,4-三嗪(4m): 浅黄色针状晶体, 收率 49.8\%. m.p. 231.2 232.8 ${ }^{\circ} \mathrm{C} ;{ }^{1} \mathrm{H}$ NMR (400 MHz, DMSO- $d_{6}$ ) $\delta: 5.51$ (s, 2H, $\left.\mathrm{CH}_{2} \mathrm{O}\right), 6.09$ (s, 2H, $\left.\mathrm{NCH}_{2}\right), 6.77$ (d, $J=8.4 \mathrm{~Hz}$, $1 \mathrm{H}, \mathrm{ArH}), 6.83$ (s, 1H, ArH), 6.93 (d, $J=8.0 \mathrm{~Hz}, 1 \mathrm{H}$, ArH), 7.18 (t, $J=8.2 \mathrm{~Hz}, 1 \mathrm{H}, \mathrm{ArH}), 7.23 \sim 7.35$ (m, 2H, BZM-H-5, H-6), 7.65 (d, $J=6.8$ Hz, 1H, BZM-H-7), 7.72 (d, $J=6.8 \mathrm{~Hz}, 1 \mathrm{H}$, BZM-H-4), 7.81 (d, $J=8.8 \mathrm{~Hz}, 2 \mathrm{H}$, Ar'H), 8.08 (d, $\left.J=8.4 \mathrm{~Hz}, 2 \mathrm{H}, \operatorname{Ar}^{\prime} \mathrm{H}\right), 9.28$ (s, 1H, triazine-H); IR (KBr) v: 3064, 2928, 2864, 1626, 1596, $1480,1462,1234,1040 \mathrm{~cm}^{-1}$. Anal. calcd for $\mathrm{C}_{24} \mathrm{H}_{17} \mathrm{Br}-$ $\mathrm{ClN}_{5} \mathrm{O}: \mathrm{C} 56.88, \mathrm{H} 3.38, \mathrm{~N} 13.82$; found C 57.05, H 3.58, $\mathrm{N} 13.96$.

3-[2-(3-氯苯氧甲基)苯并咪唑-1-亚甲基]-6-(4-氯苯 基)-1,2,4-三嗪(4n): 浅黄色固体，收率 48.9\%. m.p. $219.0 \sim 220.1{ }^{\circ} \mathrm{C} ;{ }^{1} \mathrm{H}$ NMR (400 MHz, DMSO- $\left.d_{6}\right) \delta: 5.51$ $\left(\mathrm{s}, 2 \mathrm{H}, \mathrm{CH}_{2} \mathrm{O}\right), 6.10\left(\mathrm{~s}, 2 \mathrm{H}, \mathrm{NCH}_{2}\right), 6.77(\mathrm{~d}, J=8.8 \mathrm{~Hz}$, $1 \mathrm{H}, \mathrm{ArH}), 6.83$ (s, 1H, ArH), 6.93 (d, $J=7.6 \mathrm{~Hz}, 1 \mathrm{H}$, ArH), 7.18 (t, $J=8.0 \mathrm{~Hz}, 1 \mathrm{H}, \mathrm{ArH}), 7.23 \sim 7.35$ (m, 2H, BZM-H-5, H-6), $7.60 \sim 7.70$ (m, 3H, BZM-H-7, Ar'H×2), $7.72(\mathrm{~d}, J=8.0 \mathrm{~Hz}, 1 \mathrm{H}, \mathrm{BZM}-\mathrm{H}-4), 8.16$ (d, $J=8.4 \mathrm{~Hz}$, 2H, Ar'H), 9.29 (s, 1H, triazine-H); IR (KBr) v: 3040, 2928, 2860, 1618, 1596, 1482, 1462, 1242, $1040 \mathrm{~cm}^{-1}$. Anal. calcd for $\mathrm{C}_{24} \mathrm{H}_{17} \mathrm{Cl}_{2} \mathrm{~N}_{5} \mathrm{O}$ : C 62.35, H 3.71, N 15.15; found C 62.14, H 3.88, N 14.93.

3-[2-(3-氯苯氧甲基)苯并咪唑-1-亚甲基]-6-(4-氟苯 基)-1,2,4-三嗪(4o): 浅黄色晶体，收率 54.7\%. m.p. $166.5 \sim 167.4{ }^{\circ} \mathrm{C} ;{ }^{1} \mathrm{H}$ NMR (400 MHz, $\left.\mathrm{CDCl}_{3}\right) \delta: 5.66$ (s, $\left.2 \mathrm{H}, \mathrm{CH}_{2} \mathrm{O}\right), 5.99\left(\mathrm{~s}, 2 \mathrm{H}, \mathrm{NCH}_{2}\right), 6.90 \sim 7.00(\mathrm{~m}, 3 \mathrm{H}, \mathrm{ArH})$, $7.17(\mathrm{t}, J=7.6 \mathrm{~Hz}, 1 \mathrm{H}, \mathrm{ArH}), 7.20 \sim 7.30(\mathrm{~m}, 2 \mathrm{H}$, 
BZM-H-5, H-6), $7.30 \sim 7.37$ (m, 2H, Ar'H), 7.45 7.55 (m, 1H, BZM-H7), $7.80 \sim 7.87$ (m, 1H, BZM-H-4), 8.05 (dd, $\left.J=9.2 \mathrm{~Hz}, 5.4 \mathrm{~Hz}, 2 \mathrm{H}, \mathrm{Ar}^{\prime} \mathrm{H}\right), 8.80$ (s, 1H, triazine-H); IR (KBr) v: 3064, 3037, 2929, 2858, 1596, 1482, 1461, $1233,1038 \mathrm{~cm}^{-1}$. Anal. calcd for $\mathrm{C}_{24} \mathrm{H}_{17} \mathrm{ClFN}_{5} \mathrm{O}: \mathrm{C} 64.65$, $\mathrm{H} 3.84, \mathrm{~N} 15.71$; found C 64.52, H 3.73, N 15.62.

3-[2-(3-氯苯氧甲基)苯并咪唑-1-亚甲基]-6-(3-硝基 苯基)-1,2,4-三嗪(4p): 米黄色固体, 收率 42.4\%. m.p. 156.2 157.2 ${ }^{\circ} \mathrm{C} ;{ }^{1} \mathrm{H}$ NMR (400 MHz, DMSO- $\left.d_{6}\right) \delta: 5.52$ (s, 2H, $\left.\mathrm{CH}_{2} \mathrm{O}\right), 6.14$ (s, 2H, $\left.\mathrm{NCH}_{2}\right), 6.79$ (d, J=8.4 Hz, $1 \mathrm{H}, \mathrm{ArH}), 6.81$ (s, 1H, ArH), 6.92 (d, J=7.6 Hz, 1H, $\operatorname{ArH}), 7.18$ (t, $J=8.2 \mathrm{~Hz}, 1 \mathrm{H}, \operatorname{ArH}), 7.23 \sim 7.35$ (m, 2H, BZM-H-5, H-6), 7.68 (d, J=7.2 Hz, 1H, BZM-H-7), 7.73 (d, $J=8.0 \mathrm{~Hz}, 1 \mathrm{H}$, BZM-H-4), 7.90 (t, $J=8.2 \mathrm{~Hz}, 1 \mathrm{H}$, Ar'H), 8.43 (d, $\left.J=8.0 \mathrm{~Hz}, 1 \mathrm{H}, \mathrm{Ar}^{\prime} \mathrm{H}\right), 8.56$ (d, $J=7.6 \mathrm{~Hz}$, $\left.1 \mathrm{H}, \mathrm{Ar}^{\prime} \mathrm{H}\right), 8.93$ (s, 1H, Ar'H), 9.43 (s, 1H, triazine-H); IR (KBr) v: 3086, 2945, 2869, 1618, 1596, 1531, 1477, 1461, $1352,1233,1033 \mathrm{~cm}^{-1}$. Anal. calcd for $\mathrm{C}_{24} \mathrm{H}_{17} \mathrm{ClN}_{6} \mathrm{O}_{3}: \mathrm{C}$ 60.96, H 3.62, N 17.77; found C 61.14, H 3.85, N 17.66.

3-[2-(3-氯苯氧甲基)苯并咪唑-1-亚甲基]-6-(4-硝基 苯基)-1,2,4-三嗪(4q): 黄绿色固体, 收率 48.3\%. m.p. 204.5 205.4 ${ }^{\circ} \mathrm{C} ;{ }^{1} \mathrm{H}$ NMR (400 MHz, DMSO- $d_{6}$ ) $\delta: 5.52$ (s, 2H, $\left.\mathrm{CH}_{2} \mathrm{O}\right), 6.15\left(\mathrm{~s}, 2 \mathrm{H}, \mathrm{NCH}_{2}\right), 6.76(\mathrm{~d}, J=8.4 \mathrm{~Hz}$, $1 \mathrm{H}, \operatorname{ArH}), 6.83$ (s, 1H, ArH), 6.93 (d, $J=6.8 \mathrm{~Hz}, 1 \mathrm{H}$, ArH), $7.18(\mathrm{t}, J=8.0 \mathrm{~Hz}, 1 \mathrm{H}, \mathrm{ArH}), 7.22 \sim 7.35(\mathrm{~m}, 2 \mathrm{H}$, BZM-H-5, H-6), 7.67 (d, $J=6.8$ Hz, 1H, BZM-H-7), 7.73 (d, $J=6.4 \mathrm{~Hz}, 1 \mathrm{H}$, BZM-H-4), 8.40 (d, $J=9.2 \mathrm{~Hz}, 2 \mathrm{H}$, Ar'H), 8.43 (d, $\left.J=9.6 \mathrm{~Hz}, 2 \mathrm{H}, \mathrm{Ar}^{\prime} \mathrm{H}\right), 9.39$ (s, 1H, triazine-H); IR (KBr) v: 3072, 2940, 2868, 1596, 1522, 1482, 1462, 1350, 1230, $1034 \mathrm{~cm}^{-1}$. Anal. calcd for $\mathrm{C}_{24} \mathrm{H}_{17} \mathrm{ClN}_{6} \mathrm{O}_{3}$ : C 60.96, $\mathrm{H}$ 3.62, N 17.77; found $\mathrm{C} 60.71$, H 3.81, N 18.01.

3-[2-(3-氯苯氧甲基)苯并咪唑-1-亚甲基]-6-联苯基1,2,4-三嗪(4r): 浅黄色粉末, 收率 44.4\%. m.p. 221.2 $222.1{ }^{\circ} \mathrm{C} ;{ }^{1} \mathrm{H}$ NMR (400 MHz, DMSO- $\left.d_{6}\right) \delta: 5.52$ (s, 2H, $\left.\mathrm{CH}_{2} \mathrm{O}\right), 6.10$ (s, $\left.2 \mathrm{H}, \mathrm{NCH}_{2}\right), 6.78$ (d, $\left.J=8.4 \mathrm{~Hz}, 1 \mathrm{H}, \mathrm{ArH}\right)$, 6.87 (s, 1H, ArH), 6.95 (d, J=7.6 Hz, 1H, ArH), 7.20 (t, $J=8.4 \mathrm{~Hz}, 1 \mathrm{H}, \mathrm{ArH}), 7.23 \sim 7.35$ (m, 2H, BZM-H-5, H-6), 7.42 (t, $\left.J=7.6 \mathrm{~Hz}, 1 \mathrm{H}, \mathrm{Ar}^{\prime} \mathrm{H}\right), 7.51$ (t, $J=7.4 \mathrm{~Hz}, 2 \mathrm{H}$, Ar'H), 7.67 (d, $J=7.6 \mathrm{~Hz}, 1 \mathrm{H}, \mathrm{BZM}-\mathrm{H}-7), 7.72$ (d, $J=7.2$ Hz, 1H, BZM-H-4), 7.78 (d, J=7.6 Hz, 2H, Ar'H), 7.91 (d, $\left.J=8.4 \mathrm{~Hz}, 2 \mathrm{H}, \mathrm{Ar}^{\prime} \mathrm{H}\right), 8.24$ (d, $\left.J=8.0 \mathrm{~Hz}, 2 \mathrm{H}, \mathrm{Ar}^{\prime} \mathrm{H}\right)$, 9.33 (s, 1H, triazine-H); IR (KBr) v: 3059, 3031, 2934, 2869, 1596, 1585, 1466, 1228, $1033 \mathrm{~cm}^{-1}$. Anal. calcd for $\mathrm{C}_{30} \mathrm{H}_{22} \mathrm{ClN}_{5} \mathrm{O}$ : C 71.49, H 4.40, N 13.90; found C 71.29, H
4.25, N 14.06.

3-[2-(3-氯苯氧甲基)苯并咪唑-1-亚甲基]-6-(2-荟 基)-1,2,4-三嗪(4s)：淡黄色晶体，收率 52.3\%. m.p. $210.1 \sim 211.0{ }^{\circ} \mathrm{C} ;{ }^{1} \mathrm{H}$ NMR (400 MHz, DMSO- $\left.d_{6}\right) \delta: 5.53$ $\left(\mathrm{s}, 2 \mathrm{H}, \mathrm{CH}_{2} \mathrm{O}\right), 6.12\left(\mathrm{~s}, 2 \mathrm{H}, \mathrm{NCH}_{2}\right), 6.80(\mathrm{~d}, J=7.9 \mathrm{~Hz}$, $1 \mathrm{H}, \mathrm{ArH}), 6.87$ (s, 1H, ArH), 6.94 (d, $J=8.0 \mathrm{~Hz}, 1 \mathrm{H}$, ArH), 7.18 (t, $J=8.2 \mathrm{~Hz}, 1 \mathrm{H}, \mathrm{ArH}), 7.23 \sim 7.35(\mathrm{~m}, 2 \mathrm{H}$, BZM-H-5, H-6), $7.57 \sim 7.65$ (m, 2H, Ar'H), 7.68 (d, $J=$ $8.0 \mathrm{~Hz}, 1 \mathrm{H}, \mathrm{BZM}-\mathrm{H}-7), 7.73$ (d, $J=8.0 \mathrm{~Hz}, 1 \mathrm{H}, \mathrm{BZM}-$ H-4), 8.02 (d, $\left.J=7.6 \mathrm{~Hz}, 1 \mathrm{H}, \mathrm{Ar}^{\prime} \mathrm{H}\right), 8.07$ (d, $J=9.2 \mathrm{~Hz}$, 1H, Ar'H), 8.12 (d, $\left.J=8.0 \mathrm{~Hz}, 1 \mathrm{H}, \mathrm{Ar}^{\prime} \mathrm{H}\right), 8.26$ (d, $J=8.8$ $\left.\mathrm{Hz}, 1 \mathrm{H}, \mathrm{Ar}{ }^{\prime} \mathrm{H}\right), 8.76$ (s, 1H, Ar'H), 9.43 (s, 1H, triazine-H); IR (KBr) v: 3053, 2939, 2858, 1629, 1591, 1471, 1233, $1022 \mathrm{~cm}^{-1}$. Anal. calcd for $\mathrm{C}_{28} \mathrm{H}_{20} \mathrm{ClN}_{5} \mathrm{O}: \mathrm{C} 70.36, \mathrm{H} 4.22$, N 14.65; found C 70.13, H 4.14, N 14.58.

3-[2-(3-氯苯氧甲基)苯并咪唑-1-亚甲基]-6-香豆素 基-1,2,4-三嗪(4t): 褐色固体，收率 32.6\%. m.p. 190.5 $191.9{ }^{\circ} \mathrm{C} ;{ }^{1} \mathrm{H}$ NMR (400 MHz, DMSO- $\left.d_{6}\right) \delta: 5.51(\mathrm{~s}, 2 \mathrm{H}$, $\left.\mathrm{CH}_{2} \mathrm{O}\right), 6.13$ (s, 2H, $\mathrm{NCH}_{2}$ ), 6.77 (d, $\left.J=9.2 \mathrm{~Hz}, 1 \mathrm{H}, \mathrm{ArH}\right)$, $6.86(\mathrm{~s}, 1 \mathrm{H}, \mathrm{ArH}), 6.95$ (d, $J=8.0 \mathrm{~Hz}, 1 \mathrm{H}, \mathrm{ArH}), 7.21$ (t, $J=8.0 \mathrm{~Hz}, 1 \mathrm{H}, \mathrm{ArH}), 7.33 \sim 7.45$ (m, 2H, BZM-H-5, H-6), 7.47 (t, $\left.J=8.0 \mathrm{~Hz}, 1 \mathrm{H}, \mathrm{Ar}^{\prime} \mathrm{H}\right), 7.50 \sim 7.57$ (m, 2H, BZMH-7, Ar'H), 7.66 (d, J=8.0 Hz, 1H, BZM-H-4), 7.74 (t, $\left.J=8.8 \mathrm{~Hz}, 1 \mathrm{H}, \mathrm{Ar}^{\prime} \mathrm{H}\right), 7.97$ (d, $\left.J=8.0 \mathrm{~Hz}, 1 \mathrm{H}, \mathrm{Ar}^{\prime} \mathrm{H}\right), 8.92$ (s, 1H, Ar'H), 9.26 (s, 1H, triazine-H); IR (KBr) v: 3064, 2924, 2856, 1732, 1614, 1596, 1572, 1462, 1240, 1028 $\mathrm{cm}^{-1}$. Anal. calcd for $\mathrm{C}_{27} \mathrm{H}_{18} \mathrm{ClN}_{5} \mathrm{O}_{3}$ : C 65.39, H 3.66, N 14.12; found C 65.55, H 3.79, N 14.02.

\subsection{3 目标化合物生物活性测试}

$\mathrm{Cdc} 25 \mathrm{~B}$ 是 $\mathrm{Cdc} 25$ 磷酸酯酶家族的一员. 文献报道 显示，在人类许多癌症中是过度表达的，虽然其在癌症 发生中的角色尚未被确定，但寻找到特异高效的抑制剂 对癌症研究可提供新的工具和手段. PTP1B 是第一个被 鉴定的蛋白酪氨酸磷酸酯酶，它通过对胰岛素受体的脱 磷酰化，进而在调节胰岛素敏感性和脂肪代谢过程中起 着非常重要的作用. 因而, 选择性的、高活性的 PTP1B 抑制剂在糖尿病和肥胖症的治疗中有重要的价值.

目标化合物对 Cdc25B 和 PTP1B 的抑制活性篮选实 验方法：按照参考文献[29, 30]的方法进行.

\section{References}

[1] Diana, P.; Martorana, A.; Barraja, P.; Lauria, A.; Montalbano, A.; Almerico, A. M.; Dattolo G.; Cirrincione, G. Bioorg. Med. Chem. 2007, 15, 343.

[2] Mullick, P.; Khan, S. A.; Begum, T.; Verma, S.; Kaushik, D.; Alam, O. Acta Pol. Pharm. Drug. Res. 2009, 66, 379.

[3] Irannejad, H.; Amini, M.; Khodagholi, F.; Ansari, N.; Tusi, S. K.; 
Sharifzadeh, M.; Shafiee, A. Bioorg. Med. Chem. 2010, 18, 4224

[4] Sangshetti, J. N.; Shinde, D. B. Bioorg. Med. Chem. Lett. 2010, 20, 742.

[5] Ulomskii, E. N.; Shestakova, T. S.; Deev, S. L.; Chupakhin, O. N. Russ. Chem. Bull., Int Ed. 2005, 54, 726.

[6] Guertin, K. R.; Setti, L.; Qi, L.; Dunsdon, R. M.; Dymock, B. W.; Jones, P. S.; Overton, H.; Taylor, M.; Williams, G.; Sergi, J. A.; Wang, K.; Peng, Y.; Renzetti, M.; Boyce, R.; Falcioni, F.; Garippa, R.; Olivier, A. R. Bioorg. Med. Chem. Lett. 2003, 13, 2895.

[7] Yang, H. W.; Xue, F. Q.; Zhang, L. F.; Fei, C. Z.; Zhang, Y. Chin. J. Vet. Drug. 2009, 43, 5.

[8] Branowska, D.; Karczmarzyk, Z.; Rykowski, A.; Wysocki, W.; Olender, E.; Urbańczyk-Lipkowska, Z.; Kalicki, P. J. Mol. Struct. 2010, 979, 186.

[9] Lindsley, C. W.; Wisnoski, D. D.; Wang, Y.; Leister, W. H.; Zhao, Z. J. Tetrahedron Lett. 2003, 44, 4495.

[10] Li, Y. J.; Liu, L. J.; Jin, K.; Sun, S. Q.; Xu, Y. T. Acta Chim. Sinica 2010, 68, 1577 (in Chinese).

(李英俊, 刘丽军, 靳焜, 孙淑琴, 许永廷, 化学学报, 2010, 68, 1577.)

[11] Shingalapur, R. V.; Hosamani, K. M.; Keri, R. S.; Hugar, M. H. Eur. J. Med. Chem. 2010, 45, 1753.

[12] Gaba, M.; Singh, D.; Singh, S.; Sharma, V.; Gaba, P. Eur. J. Med. Chem. 2010, 45, 2245.

[13] Goulet, S.; Poupart, M. A.; Gillard, J.; Poirier, M.; Kukolj, G.; Beaulieu, P. L. Bioorg. Med. Chem. Lett. 2010, 20, 196.

[14] Ansari, K. F.; Lal, C. Eur. J. Med. Chem. 2009, 44, 2294.

[15] Ansari, K. F.; Lal, C. Eur. J. Med. Chem. 2009, 44, 4028.

[16] Saraswathi, T. V.; Srinivasan, V. R. Tetrahedron Lett. 1971, 25,
2315.

[17] Saraswathi, T. V.; Srinivasan, V. R. Tetrahedron 1977, 33, 1043.

[18] Misra, U.; Hitkari, A.; Saxena, A. K.; Gurtu, S.; Shanker, K. Eur. J. Med. Chem. 1996, 31, 629.

[19] Phucho, T.; Nongpiur, A.; Tumtin, S.; Nongrum, R.; Myrboh, B.; Nongkhlaw, R. L. ARKIVOC 2008, (xv), 79.

[20] Musatov, D. M.; Kurilov, D. V.; Rakishev, A. K. Ukr. Bioorg. Acta 2008, 1,61 .

[21] Sun, Y. F.; Pan, W. L.; Wu, R. T.; Song, H. C. Chin. J. Org. Chem 2006, 26, 1079.

[22] Thirumurugan, P.; Perumal, P. T. Dyes Pigm. 2011, 88, 403.

[23] Ghazvini Zadeh, E. H.; El-Gendy, B. E. M.; Popa, A. G.; Katritzky, A. R. MedChemComm 2012, 3, 52.

[24] Kidwai, M.; Sapra, P.; Bhushan, K. R.; Misra, P. Synth. Commun. 2001, 31, 1639.

[25] Bäurle, S.; Blume, T.; Günther, J.; Henschel, D.; Hillig, R. C.; Husemann, M.; Mengel, A.; Parchmann, C.; Schmid, E.; Skuballa, W. Bioorg. Med. Chem. Lett. 2004, 14, 1673.

[26] Lessard, L.; Stuible, M.; Tremblay, M. L. Biochim. Biophys. Acta 2010, 1804, 613.

[27] Li, Y. J.; Sun, Y. Z.; Xu, Y. T.; Jin, K.; Wen, L. P.; Hou, N. B.; Sun, X. X. Chin. Chem. Lett. 2007, 18, 1047.

[28] Li, Y. J.; Liu, L. J.; Jin, K.; Xu, Y. T.; Sun, S. Q. Chin. Chem. Lett. 2010, 21, 293.

[29] Huang, W. G.; Jiang, Y. Y.; Li, Q.; Li, J.; Li, J. Y.; Lu, W.; Cai, J. C. Tetrahedron 2005, 61, 1863.

[30] Sun, L. P.; Shen, Q.; Piao, H. H.; Ma, W. P.; Gao, L. X.; Zhang, W.; Nan, F. J.; Li, J.; Piao, H. R. Eur. J. Med. Chem. 2011, 46, 3630 .

(Li, L.; Lu, Z.) 